\title{
Prevalencia de asma y sus síntomas en población escolar de Cuernavaca, Morelos, México
}

\author{
Eleazar Mancilla-Hernández, ${ }^{1}$ Evaristo Víctor Manuel González-Solórzano, ${ }^{2}$ Miguel Ángel Medina-Ávalos, ${ }^{3}$ \\ Raúl Humberto Barnica-Alvarado ${ }^{4}$
}

\begin{abstract}
Background: Asthma is a chronic disease that has increased over the past 2 decades.

Objective: To determine the prevalence of asthma and its symptoms in the city of Cuernavaca, Morelos, Mexico.

Methods: A descriptive study of prevalence in public schools of the city of Cuernavaca, Morelos, at the preschool, elementary, and high school levels using the "Asthma diagnosis for epidemiological studies questionnaire."

Results: 7947 surveys were obtained from students ages 3 to 15 years, funding a prevalence of asthma of $11.9 \%, 49 \%$ in boys and $51 \%$ in girls. As for the symptoms of asthma, the highest prevalence was cough that increases with cold weather $(63 \%)$, however, in asthma patients this symptom only occurred in $17 \%$; chest tightness had the lowest prevalence of symptoms (10\%); however, in asthma patients it was $49 \%$.

Conclusions: The asthma diagnosis for epidemiological studies questionnaire found an average asthma prevalence of $11.9 \%$ in preschool, elementary, and high school students, slightly below that diagnosed in other cities in central Mexico. The prevalence of asthma symptoms was higher than the diagnosis of asthma.
\end{abstract}

Keywords: Asthma; Prevalence; Symptoms; Epidemiological studies

Este artículo debe citarse como: Mancilla-Hernández E, González-Solórzano EVM, Medina-Ávalos MÁ, BarnicaAlvarado RH. Prevalencia de asma y sus síntomas en población escolar de Cuernavaca, Morelos, México. Rev Alerg Mex. 2016;63(4):351-357

${ }^{1}$ Centro de Investigación en el Área de la Salud A.C. Puebla,

Puebla

${ }^{2}$ Práctica privada. Cuernavaca, Morelos

${ }^{3}$ Instituto de Seguridad y Servicios Sociales de los Trabajadores del Estado, Hospital Regional Veracruz, Servicio de Alergia. Veracruz, Veracruz

${ }^{4}$ Práctica privada. Ciudad Obregón, Sonora, México

\begin{abstract}
Correspondencia: Eleazar Mancilla-Hernández. manele05@yahoo.com.mx
\end{abstract}

Recibido: 2016-05-25

Aceptado: 2016-08-19 


\title{
Resumen
}

Antecedentes: El asma es una enfermedad crónica que se ha incrementado en las 2 últimas décadas.

Objetivo: Determinar la prevalencia de asma y sus síntomas en la ciudad de Cuernavaca, Morelos, México.

Métodos: Estudio descriptivo de prevalencia en escuelas oficiales de la ciudad de Cuernavaca, Morelos, de nivel preescolar, primaria y secundaria, mediante el "Cuestionario diagnóstico de asma para estudios epidemiológicos".

Resultados: Se obtuvieron 7947 encuestas en escolares de 3 a 15 años, encontrándose una prevalencia de asma de $11.9 \%, 49 \%$ en niños y $51 \%$ en niñas. En cuanto a los síntomas de asma, la mayor prevalencia la obtuvo la tos que aumenta con el frío (63\%), sin embargo, en los pacientes con asma este síntoma solo se presentó en 17\%; la opresión torácica tuvo la prevalencia más baja entre los síntomas (10\%), sin embargo, en pacientes con asma fue de $49 \%$.

Conclusiones: Con el cuestionario diagnóstico de asma para estudios epidemiológicos se encontró una prevalencia promedio de asma de $11.9 \%$ en preescolares, escolares y alumnos de secundaria, discretamente inferior a la diagnosticada en otras ciudades del centro de México. La prevalencia de los síntomas de asma resultó más elevada que la del diagnóstico de asma.

Palabras clave: Asma; Prevalencia; Síntomas; Estudios epidemiológicos

\author{
Abreviaturas y siglas \\ ISAAC, The International Study of Asthma and Allergies \\ in Childhood
}

\section{Introducción}

El asma es una de las enfermedades crónicas más frecuentes en los niños; ${ }^{1}$ es un problema de salud pública que se ha incrementado en las últimas 2 décadas, ${ }^{2}$ principalmente en los países desarrollados. ${ }^{3}$ La mayoría de los estudios de prevalencia se ha realizado con un instrumento validado en la década de 1990 por el ISAAC (The International Study of Asthma and Allergies in Childhood), con la finalidad de optimar el análisis epidemiológico del asma, la rinoconjuntivitis y la dermatitis atópica. ${ }^{4}$

Con ese instrumento se han encontrado grandes variaciones en la prevalencia de esas enfermedades entre países y regiones e, incluso, entre ciudades próximas. ${ }^{5,6} \mathrm{El}$ ISAAC mostró una mayor prevalencia de asma en los países industrializados, hasta de $30 \%$.

Según el ISSAC, en América Latina la prevalencia varía de 5.7 a $16.5 \%{ }^{7}$ Un análisis realizado en Cuernavaca, Morelos, México, mostró una prevalencia actual de asma de $6.6 \%$ en adolescentes de 13 y 14 años; ${ }^{7}$ en Acapulco, Guerrero, en escolares se obtuvo $33 \% .{ }^{8}$ Con el empleo del instrumento validado "Cuestionario diagnóstico de asma para estudios epidemiológicos" (Anexo 1), ${ }^{9}$ en alumnos escolares y adolescentes se encontró una prevalencia de 14\% en Puebla, Puebla; $17 \%$ en Tulancingo, Hidalgo; 14\% en Cancún, Quintana Roo; y 7\% en Tlaxcala, Tlaxcala. ${ }^{10}$

En Brasil se registra una prevalencia alta de asma: $24.3 \%$ en niños y $19 \%$ en adolescentes. ${ }^{11}$ Con la pregunta correspondiente a asma actual del instrumento del ISAAC, al evaluar la prevalencia de asma en zonas urbana, semiurbana y rural de Chile se encontró 16,10 y $6 \%$, respectivamente, en adolescentes de 13 a 14 años. ${ }^{12}$ En Buenos Aires, Argentina, se informa una prevalencia de asma de $15.4 \%$ en niños entre 6 y 7 años y de $9.9 \%$ en adolescentes entre 13 y 14 años. ${ }^{13}$

Considerando que la mayor parte de los estudios de prevalencia en asma se basan en realidad en la prevalencia de algún síntoma, el objetivo de la 
investigación que se presenta fue comparar la prevalencia de cada síntoma con la prevalencia del diagnóstico de asma basado en un cuestionario que considera antecedentes, síntomas de asma, síntomas de hiperreactividad bronquial y signos de exploración, y determinar si existen diferencias entre una y otra.

\section{Métodos}

Se realizó un estudio descriptivo de prevalencia en Cuernavaca, Morelos, México, en escuelas oficiales autorizadas por la Secretaría de Educación Pública del estado. La asignación de las escuelas para las encuestas fue realizada por esa misma Secretaría. Con previa autorización de los padres se aplicó el "Cuestionario diagnóstico de asma para estudios epidemiológicos”. El estudio se realizó de junio a diciembre de 2015.

\section{Análisis estadístico}

Se obtuvieron promedios, desviación estándar en la edad y grupos de edad, porcentajes e intervalos de confianza para una proporción en la prevalencia de los síntomas de asma y porcentaje en la prevalencia del diagnóstico de asma.

\section{Resultados}

Se aplicaron 7947 encuestas, 3875 a sujetos del sexo masculino y a 4072 del sexo femenino; las edades fluctuaron entre 3 y 15 años de edad, obteniéndose un promedio de $8.4 \pm 4.3$ años. La prevalencia de asma encontrada en Cuernavaca, Morelos, fue de $11.9 \% ; 49 \%$ correspondió al sexo masculino y $51 \%$ al sexo femenino.
En la Figura 1 se muestra el porcentaje de asma por edades y la fluctuación del diagnóstico del asma que osciló de 15 a $9 \%$ de los 3 a 15 años de edad. Se apreciaron niveles similares de prevalencia de asma en los distintos grupos de edad: $11.8 \%$ en los preescolares (entre 3 y 5 años), $11.5 \%$ en los escolares (de 6 a 12 años) y $12.7 \%$ en los alumnos de secundaria (de 13 a 15 años).

Respecto a la prevalencia de los síntomas (Cuadro 1) se identificó una amplia variación entre ellos: el que obtuvo el valor más alto fue la tos que aumenta con el frío $(63 \%)$ y el de menor, la opresión del pecho (10\%).

En el Cuadro 2 se compara la prevalencia de los síntomas de asma en relación con la prevalencia de asma en Cuernavaca, Morelos, México: prácticamente en todos los casos fue mayor. Los síntomas con más alta frecuencia en el asma correspondieron a sibilancias, opresión torácica, dificultad respiratoria y tos recurrente, en orden descendente.

\section{Discusión}

La importancia de investigar la prevalencia de asma y de cada síntoma en una misma muestra de 7947 escolares radica en que la mayoría de los estudios publicados que determinan la prevalencia de asma en realidad indican la prevalencia de algún síntoma; de estos, los más frecuentes son las sibilancias en los últimos 12 meses y en alguna ocasión. Con el cuestionario empleado, el cual consta de 9 preguntas con asignación de valores, se apreció que la prevalencia de cada uno de los síntomas resultó diferente a la del asma.

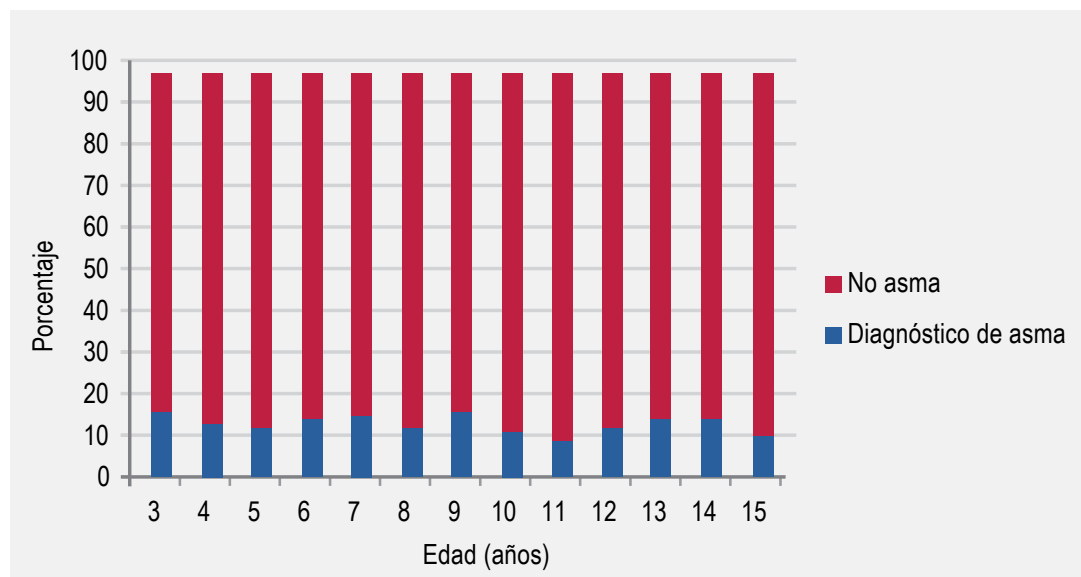

Figura 1. Porcentaje de asma por grupos de edad 


\begin{tabular}{|c|c|c|c|c|c|c|}
\hline Síntomas & & Encuestados & $\begin{array}{l}\text { Diagnóstico } \\
\text { de asma }\end{array}$ & No asma & $\begin{array}{c}\text { Prevalencia } \\
(\%)\end{array}$ & IC 95\% \\
\hline \multirow[t]{2}{*}{ Tos recurrente } & Sí & 1901 & 636 & 1265 & 24 & $22.9-25$ \\
\hline & No & 6046 & 313 & 5733 & & \\
\hline Tos que aumenta con el frío & Sí & 4991 & 865 & 4126 & 63 & $61.9-64$ \\
\hline \multirow{3}{*}{$\begin{array}{l}\text { Tos que aumenta con el ejercicio } \\
\text { físico }\end{array}$} & No & 2956 & 84 & 2872 & & \\
\hline & Sí & 2489 & 580 & 1909 & 31 & 29.9-32 \\
\hline & No & 5458 & 369 & 5089 & & \\
\hline \multirow[t]{2}{*}{ Tos de predominio nocturno } & Sí & 2785 & 609 & 2176 & 35 & $33.9-36.04$ \\
\hline & No & 5162 & 340 & 4822 & & \\
\hline \multirow[t]{2}{*}{ Sibilancias recurrentes } & Sí & 1328 & 941 & 387 & 16.7 & $15.8-17.5$ \\
\hline & No & 6619 & 8 & 6611 & & \\
\hline \multirow[t]{2}{*}{ Dificultad respiratoria } & Sí & 1142 & 500 & 642 & & \\
\hline & No & 6805 & 449 & 6356 & 14 & $13.2-14.76$ \\
\hline \multirow[t]{2}{*}{ Opresión del pecho } & Sí & 781 & 388 & 393 & 10 & $9.34-10.65$ \\
\hline & No & 7166 & 561 & 6605 & & \\
\hline \multirow[t]{2}{*}{ Cuadros catarrales recurrentes } & Sí & 3349 & 468 & 2881 & 42 & $40.9-43.08$ \\
\hline & No & 4598 & 481 & 4117 & & \\
\hline
\end{tabular}

La prevalencia de asma en Cuernavaca, Morelos, obtenida en 2015 con el "Cuestionario diagnóstico de asma para estudios epidemiológicos" fue de $11.9 \%$ en preescolares, escolares y adolescentes, superior a 6.6\% informado en escolares en 1998 y a $8.09 \%$ en escolares y adolescentes en 2002 ; en los dos últimos casos con la pregunta de sibilancias en los últimos 12 meses. $^{14}$

Respecto a los síntomas que habitualmente manifiestan el asma, la prevalencia de algunos fue muy superior a la del asma, como sucedió con la tos recurrente, la tos que aumenta con el frío, la tos que se produce con el ejercicio físico y la tos de predomino nocturno. Los síntomas que más se acercaron a la prevalencia de asma fueron opresión torácica, $10 \%$; dificultad respiratoria, $14 \%$; y sibilancias, $16 \%$.

Lo anterior permite considerar que la prevalencia de los síntomas no parece ser un buen indicador de la prevalencia de asma.

El "Cuestionario diagnóstico para prevalencia de asma" se ha utilizado en la determinación de prevalencias en Puebla (Puebla), Tulancingo (Hidalgo), Tlaxcala (Tlaxcala) y Cancún (Quintana Roo), lugares en los que no hay información anterior respecto al diagnóstico de asma con base en la prevalencia de algún síntoma, de ahí que no puedan formularse comparaciones.

Aun no contamos con estudios publicados de prevalencia de asma en los cuales en una misma población se utilice el "Cuestionario diagnóstico para prevalencia de asma" y cuestionarios que se basen en la prevalencia de un síntoma; sin embargo, el instrumento que utilizamos incluye la pregunta que indaga la manifestación de sibilancias recurrentes, síntoma considerado indicativo de la presencia de asma en la mayoría de las encuestas. Nuestros resultados indican que con cuestionarios basados en un síntoma, como las sibilancias, se registran amplias variaciones en la prevalencia de asma entre regiones e, incluso, entre ciudades cercanas. ${ }^{5,6}$ Esperamos que con "Cuestionario diagnóstico para prevalencia de asma" se obtengan menores variaciones y mayor proximidad a valores reales.

Existió elevación sustancial de la prevalencia atribuible al incremento mismo de la enfermedad (por el aumento de la población y de la contaminación ambiental) o al instrumento de medición utilizado, el cual para detectar la prevalencia de asma 
Mancilla-Hernández E et al. Asma y sus síntomas en población escolar

\begin{tabular}{|l|c|c|c|c|}
\hline \begin{tabular}{l} 
Cuadro 2. Prevalencia de asma y prevalencia y frecuencia de los síntomas en pacientes asmáticos de Cuernavaca, Morelos, México \\
\hline Síntomas
\end{tabular} & $\begin{array}{c}\text { Prevalencia } \\
\text { del síntoma (\%) }\end{array}$ & $\begin{array}{c}\text { Prevalencia } \\
\text { de asma (\%) }\end{array}$ & $\begin{array}{c}\text { Frecuencia del síntoma } \\
\text { en el asma (\%) }\end{array}$ & IC 95\% \\
\hline Tos recurrente & 24 & 11.9 & 33 & $30.8-35.1$ \\
\hline Tos que aumenta con el frío & 63 & 11.9 & 17 & $15.9-18.0$ \\
\hline $\begin{array}{l}\text { Tos que aumenta } \\
\text { con el ejercicio físico }\end{array}$ & 31 & 11.9 & 23 & $21.3-24.6$ \\
\hline Tos de predominio nocturno & 35 & 11.9 & 21 & $19.4-22.5$ \\
\hline Sibilancias recurrentes & 16.7 & 11.9 & 71 & $68.5-73.4$ \\
\hline Dificultad respiratoria & 14 & 11.9 & 43 & $40-45.8$ \\
\hline Opresión del pecho & 10 & 11.9 & 49 & $45.4-52.5$ \\
\hline Cuadros catarrales recurrentes & 42 & 11.9 & 14 & $13.5-14.37$ \\
\hline
\end{tabular}

se basa en 9 preguntas que establecen una exploración más amplia de los síntomas para el diagnóstico de asma.

\section{Conclusiones}

Con el "Cuestionario diagnóstico de asma para estudios epidemiológicos" se encontró una prevalencia promedio de asma de $11.9 \%$ en los grupos de edad correspondientes a preescolares, escolares y alumnos de secundaria, y la prevalencia de los síntomas de asma en general fue diferente a la encontrada en diagnóstico de asma. Con el instrumento se identificó que los síntomas con mayor frecuencia en los pacientes con asma fueron los que tuvieron su prevalencia más cercana a la prevalencia del diagnóstico de asma.

\section{Referencias}

1. Papadopoulos NG, Arakawa H, Carlsen KH, CustovicA, Gern J, Lemanske R, et al. International consensus on (ICON) pediatric asthma. Allergy. 2012;67(8):976-997. doi: 10.1111/j.1398-9995.2012.02865.x

2. Cooper PJ, Rodrigues LC, Cruz AA, Barreto ML. Asthma in Latin America: a public heath challenge and research opportunity. Allergy. 2009;64(1):5-17. doi: 10.1111/j.1398-9995.2008.01902.x

3. García-Marcos L, Pacheco-González R. A sequel of the International Study of Asthma and Allergies in Childhood or a prelude to the Global Asthma Network? J Pediatria. 2015;91(1):1-3. doi: 10.1016/j. jped.2014.09.001

4. Burney $\mathrm{P}$, Chin S. Developing a new questionnaire for measuring the prevalence and distribution of asthma. Chest. 1987;91(6 Suppl):79S-83S.

5. Carvajal-Urueña L, García-Marcos R. Busquets-Monge M, Morales Suárez-Varela M, García de Andoine N, Batlles-Garrido J, et al. Variaciones geográficas en la prevalencia de síntomas de asma en los niños y adolescentes españoles. International Study of Asthma and Allergies in Childhood (ISAAC). Phase III España. Arch Bronconeumol. 2005;41:659-666. doi: 10.1016/S0300-2896(05)70721-3

6. Lai R, Beasley J, Crane S, Foliaki J, Shah S. Global variation in the prevalence and severity of asthma symptoms: Phase three of the International Study of Asthma and Allergies in Childhood (ISAAC). Thorax. 2009;64:476-483. doi:10.1136/thx.2008.106609

7. The International Study of Asthma and Allergies in Childhood (ISAAC) Steering Committee. Worlwide variation in prevalence of symptoms of asthma allergic rhinoconjunctivitis and atopic eczema: ISSAC. Lancet. 1998:351(9111):1225-1232.

8. Rojas-Molina N, Legorreta-Soberanis J, Olvera-Guerra F. Prevalencia de asma y factores de riesgo en municipios del estado de Guerrero, México. Rev Alerg Mex. 2001;4884):115-118. 
9. Mancilla-Hernández E, Barnica-Alvarado RH, Morfin-Maciel B, Larenas-Linneman D. Validación de un cuestionario diagnóstico de asma en niños y adultos para estudios epidemiológicos. Rev Alerg Mex. 2014;61(2):73-80. Disponible en: http://revistaalergia.mx/ojs/index.php/ram/article/download/29/42

10. Mancilla-Hernández E, Medina-Ávalos MA, Barnica-Alvarado RH, Soto-Candia D, et al. Prevalencia de asma y determinación de los síntomas como indicadores de riesgo. Rev Alerg Mex. 2015;62:271-278. Disponible en: http://revistaalergia.mx/ojs/index.php/ram/article/download/91/197

11. Solé D, Camelo-Nunes IC, Wandalsen GF, Mallozi MC, Naspitz CK, Brazilian ISAAC's Group. Is the prevalence of asthma and related symptoms among Brazilian children related to socioeconomic status. J Asthma. 2008;45(1):19-25. doi: 10.1080/0277090070149605

12. Kausel L, Boneberger A, Calvo M, Radon K. Childhood asthma and allergies inurban, semiurban, and rural residential sectors in Chile. Sci World J. 2013. ID 937935, 4 pages. doi: 10.1155/2013/937935

13. Chong Neto $\mathrm{H}$, Augusto Rosário N, Solé D. Asthma and rhinitis in South America: How different they are from other parts of the world. Allergy Asthma Immunol Res. 2012;4(2):62-67. doi: 10.4168/ aair.2012.4.2.62

14. Gutiérrez-Delgado RI, Barraza-Villarreal A, Escamilla-Nuñez MC, Solano-González M, Moreno-Macías H, Romieu I. Consumo de alimentos y asma en niños escolares de Cuernavaca, Morelos. Salud Publica Mex. 2009;51(3):202-211. Disponible en: http://www.scielo.org.mx/scielo.php?script=sci_ arttext\&pid=S0036-36342009000300010\&Ing=es 
Mancilla-Hernández E et al. Asma y sus síntomas en población escolar

Anexo 1. Cuestionario diagnóstico de asma para estudios epidemiológicos

$\begin{array}{llll}\text { Nombre: } & & \text { Fecha: } \\ \text { Edad: } & \text { Sexo:M } \square \text { F } & \text { Ciudad: } & \text { Estado: }\end{array}$

Marque con una $\mathrm{X}$ las respuestas que correspondan

1. Antecedentes familiares de alergia (asma, rinitis alérgica, urticaria, alergia a alimentos, alergia a medicamento, a picadura de insectos, dermatitis atópica)

$\square i ́$
NNo

2. Cuadros de tos recurrentes

$\square$ Sí

$\square$ No

Frecuencia de los cuadros de tos:

口Todo el tiempo $\quad \square$ Una vez al mes $\square$ Cada 6 meses

口Otro tiempo

3. La tos aumenta con el frío

$\square$ Sí $\quad \square$ No

4. La tos aumenta con ejercicio

$\square \mathrm{Si}$

$\square$ No

5. La tos es de predominio nocturno

$\square$ Sí $\square$ No

6. Sibilancias recurrentes (silbidos, "chilla" el pecho)

$\square$ Sí $\quad \square$ No

Si respondió sí:

口Una sola vez $\square 2$ Veces $\square 30$ más veces

7. Dificultad respiratoria

$\square$ Sí $\quad \square$ No

8. Opresión del pecho

$\square$ Sí

$\square$ No

9. Cuadros recurrentes de catarro con tos.

$\square$ Sí $\square$ No

Si respondió Sí:

$\square$ Siempre

$\square$ Casi siempre

$\square$ A veces

Puntuación: $1=0.1,2=0.25,3=0.1,4=0.1,5=0.1,6=0.5,7=0.1,8=0.1,9=0.1$

Diagnóstico positivo $>0.75$ (solo con puntuación) 